\title{
Random Response to Periodic Excitation with Correlated Disturbances
}

\author{
By Zhikun Hou, ${ }^{1}$ Yunshen Zhou, ${ }^{2}$ Mikhail F. Dimentberg, ${ }^{3}$ and Mohammad Noori ${ }^{4}$
}

\begin{abstract}
The paper addresses non-Gaussian stationary response of linear single-degree-of-freedom (SDOF) systems subject to a periodic excitation with correlated random amplitude and phase disturbances that are modeled as correlated Gaussian white noise processes. Correlation between amplitude and phase modulation is specified by the cross-correlation coefficient. Numerical results for the second and fourth moment responses are presented. The probability density function of the response is calculated based on the cumulant-neglect closure method. Non-Gaussian nature of the response is discussed in terms of the excess factor. The results show that the moment responses generally increase with larger random amplitude disturbance and may decrease with larger random phase modulation for a lightly damped system at resonance. The cross correlation between amplitude and phase disturbances plays an important role in the system moment response. Larger system damping results in smaller system moment responses. The moment response may approach a limiting value, depending on the intensity of the amplitude disturbance, as the relative detuning or phase modulation increases. For the case of the phase modulation alone, the response may become Gaussian in the sense of up to the fourth-order moment for sufficiently large relative detuning or random phase disturbances.
\end{abstract}

\section{INTRODUCTION}

The model of periodic excitation with random phase modulation was used by Dimentberg (1988) and Wedig (1989) in the investigation of Mathieu's-type stochastic systems. Applications of the model may be found later in studies of parametric excitation of a straight pipe due to slug flow of a twophase fluid (Dimentberg 1991), stability and subcritical dynamics of structures with spatially disordered traveling parametric excitation (Dimentberg 1992), and the stability analysis of bridges in turbulent flow ( $\mathrm{Lin}$ and $\mathrm{Li} \mathrm{1993).} \mathrm{The} \mathrm{form}$ of periodic functions with uniformly distributed random phase disturbance was also employed in simulation of earthquake ground motions (Shinozuka and Deodatis 1988). The nonGaussian nature of stationary response of SDOF systems under periodic excitation with random phase modulation was generally discussed (Dimentberg et al. 1993) where equations are derived for up to the fourth-order moments and non-Gaussian nature was studied in terms of the excess factor. Comparison of the exact solution with an approximate solution by stochastic averaging method was presented as well. Recently, the model has been extended to the nonstationary case where the nonstationary random phase disturbance was modeled as a modulated stationary process (Hou et al. 1994, 1995). Different types of envelope functions are employed in the study in order to investigate the effects of buildup and decay rate and duration of the nonstationary phase modulation. The nonstationary second- and fourth-order moments are computed by numerically solving differential equations for the transient moment. Significance of nonstationarity and nonnormality is demonstrated by some numerical results.

The model of periodic excitation with random phase modulation was originally developed to represent basically peri-

\footnotetext{
'Asst. Prof., Dept. of Mech. Engrg., Worcester Polytechnic Inst., Worcester, MA 01609.

${ }^{2}$ Grad. Student., Dept. of Mech. Engrg., Worcester Polytechnic Inst., Worcester, MA.

${ }^{3}$ Prof., Dept. of Mech. Engrg., Worcester Polytechnic Inst., Worcester, MA.

${ }^{4}$ Prof., Dept. of Mech. Engrg., Worcester Polytechnic Inst., Worcester,
}

odic phenomena with random deviations from perfect periodicity, where fluctuations of the excitation amplitude are of secondary importance as compared with those of the excitation frequency and, therefore, are ignored. As a result, the amplitude of the periodic excitation is assumed to be a constant. The assumption brings certain limitation to its applications because the excitation will be bounded within a certain range. It may not be satisfactory for many engineering problems that involve comparable fluctuations in both amplitude and phase, such as modeling of seismic ground accelerations (Grigoriu 1988) or ocean wave loading (Longuet-Higgins 1957), that may exhibit randomness in both amplitude and frequency content. In a preliminary study (Hou et al. 1995), an excitation model with uncorrelated amplitude and phase modulations was proposed. The effects of amplitude and/or phase modulations on linear SDOF system response are discussed.

To include the cross correlation between amplitude and phase disturbances into analysis to evaluate its role in the system moment response, this paper presents a general study for a periodic excitation with correlated random amplitude and phase disturbances. Application of this model to linear SDOF systems is demonstrated. Stationary moment responses of up to the fourth order are presented. The probability density function of the response is computed by the cumulant-neglect closure method. The excess factor is used as an index of nonGaussian behavior of the response. Correlation between amplitude and phase modulations is described by the crosscorrelation coefficient. The significance of amplitude disturbance and phase modulation as well as their correlation are illustrated by numerical results.

\section{FORMULATION}

The governing differential equation of motion of a linear SDOF system subjected to a random loading is written as

$$
d^{2} x / d t^{2}+2 \alpha(d x / d t)+\Omega^{2} x=y(t)
$$

where $x(t)=$ displacement response; $\Omega$ and $\alpha=$ natural frequency and damping constant of the system, respectively; and $y(t)=$ external excitation. For a periodic excitation with random amplitude and phase disturbances, $y(t)$ has a form

$$
y(t)=\left[\lambda+\xi_{1}(t)\right] \cos v ; \quad d v / d t=\mu+\xi_{2}(t)
$$

where $\lambda=$ mean amplitude; and $\mu=$ mean frequency of the periodic excitation. Both of them are constant here. $\xi_{1}(t)$ and $\xi_{2}(t)$, the amplitude and phase disturbances, are modeled as 
stationary random processes. In the present study, $\xi_{1}(t)$ and $\xi_{2}(t)$ are assumed to be correlated Gaussian white noise processes with a zero mean; intensities $D_{1}$ and $D_{2}$, respectively; and cross intensity $D_{12}$. That is

$$
\begin{array}{rr}
E\left[\xi_{1}(t)\right]=E\left[\xi_{2}(t)\right]=0 ; & E\left[\xi_{1}(t) \xi_{1}(t+\tau)\right]=D_{1} \delta(\tau) \\
E\left[\xi_{2}(t) \xi_{2}(t+\tau)\right]=D_{2} \delta(\tau) ; & E\left[\xi_{1}(t) \xi_{2}(t+\tau)\right]=D_{12} \delta(\tau)
\end{array}
$$

In $(3), \delta(\cdot)=$ Dirac's delta function; and $E[\cdot]=$ mathematical expectation operator. Obviously, the cross intensity $D_{12}$ specifies the cross correlation between $\xi_{1}(t)$ and $\xi_{2}(t)$.

The linear cross-correlation coefficient $\rho$ is introduced here to describe the cross correlation between $\xi_{1}(t)$ and $\xi_{2}(t)$. $\rho$ satisfies the following relationship:

$$
D_{12}=\rho \sqrt{D_{1} D_{2}}
$$

where $-1 \leq \rho \leq 1$. As two special cases, $\xi_{1}(t)$ and $\xi_{2}(t)$ are uncorrelated if $\rho=0$ and they are fully correlated if $\rho= \pm 1$. $\rho$ may be used as a measure of linear correlation between $\xi_{1}(t)$ and $\xi_{2}(t)$.

Some graphic samples of the excitation model expressed by (2) are presented in Fig. 1. These results are based on computer simulation. Fig. 1(a) shows time history of periodic excitation with small phase modulation but no amplitude disturbance. The phase shift caused by random phase modulation can be clearly observed. The excitation is bounded within the range $[-1,1]$. In the case of periodic excitation with very small amplitude disturbance only as shown in Fig. 1(b), there are amplitude fluctuations and the peaks and troughs of the excitation may exceed the boundaries -1 and 1 . Periodic excitations with uncorrelated, and full correlated amplitude and phase disturbances are illustrated in Fig. 1(c,d). The excita-

(a)

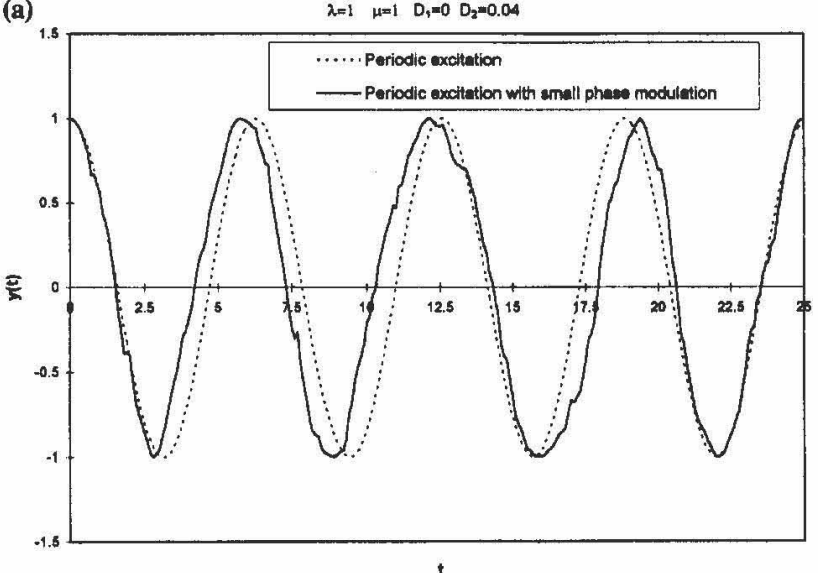

(c)

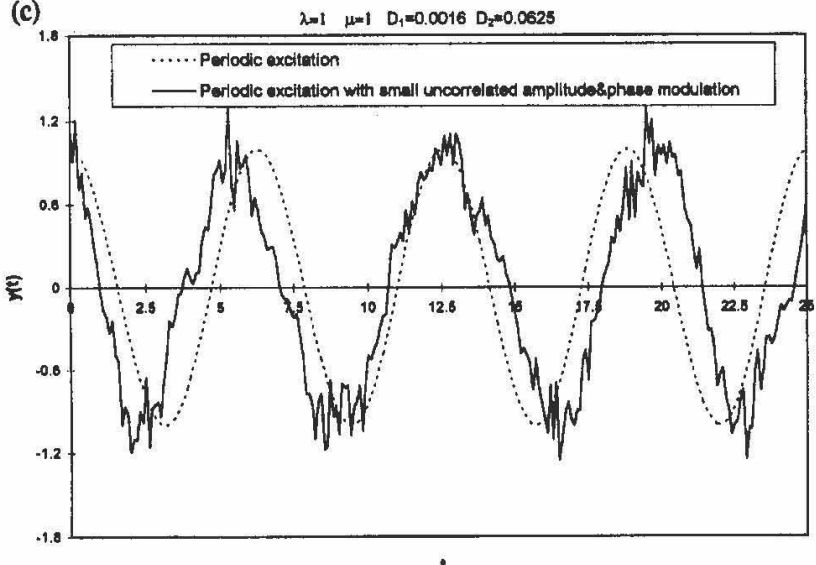

tions contain irregularities in both amplitude and phase. However, the role of cross-correlation coefficient can hardly be determined by observation.

An index of non-Gaussian nature of the response used here is the excess factor $K$ defined by

$$
K=3-E\left[x^{4}\right] /\left(E\left[x^{2}\right]\right)^{2}
$$

Both second- and fourth-order moments of the response are required in order to evaluate $K$. For a Gaussian process, the excess factor $K=0$, which is an indication of Gaussian response in the sense of up to the fourth-order moment.

The original system [(1)] subjected to the periodic excitation with random amplitude and phase disturbances expressed by (2) can be reformulated in the state space by introducing the following state variables:

$$
z_{1}=x ; \quad z_{2}=d x / d t ; \quad z_{3}=\cos v ; \quad z_{4}=\sin v \quad(6 a-d)
$$

In view of (2), the corresponding stochastic Cauchy problem for the state variables $z_{i},(i=1,2,3,4)$ may be written as a set of the following four "physical" or Stratonovich stochastic differential equations:

$$
\begin{array}{r}
d z_{1} / d t=z_{2} ; \quad d z_{2} / d t=-\Omega^{2} z_{1}-2 \alpha z_{2}+\left[\lambda+\xi_{1}(t)\right] z_{3} \\
d z_{3} / d t=-\left[\mu+\xi_{2}(t)\right] z_{4} ; \quad d z_{4} / d t=\left[\mu+\xi_{2}(t)\right] z_{3}
\end{array}
$$

Recognizing the difficulties of solving the associated Fokker-Planck equation for the probability density function of the response, this SDE set is analyzed by the method of moments. Due to the autonomous representation of trigonometric functions in (6), the set of (7) is supplemented by an obvious constraint equation

(b)

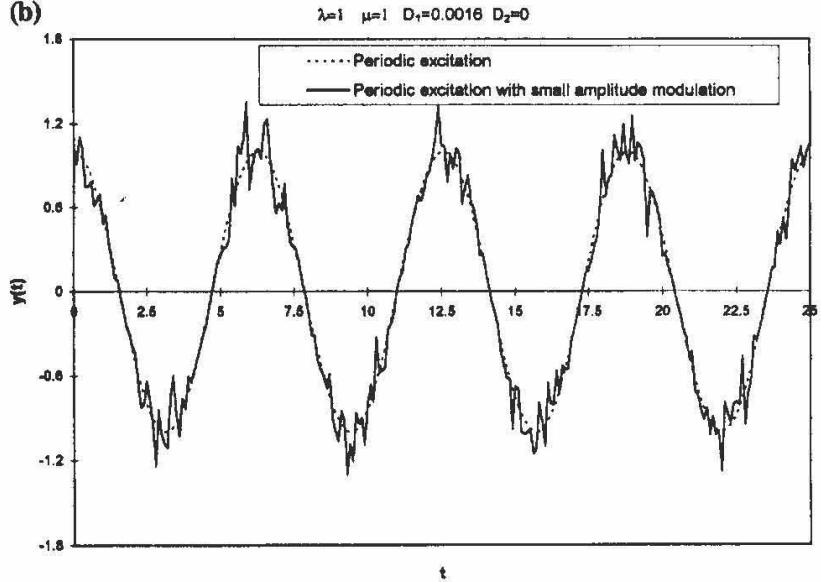

(d)

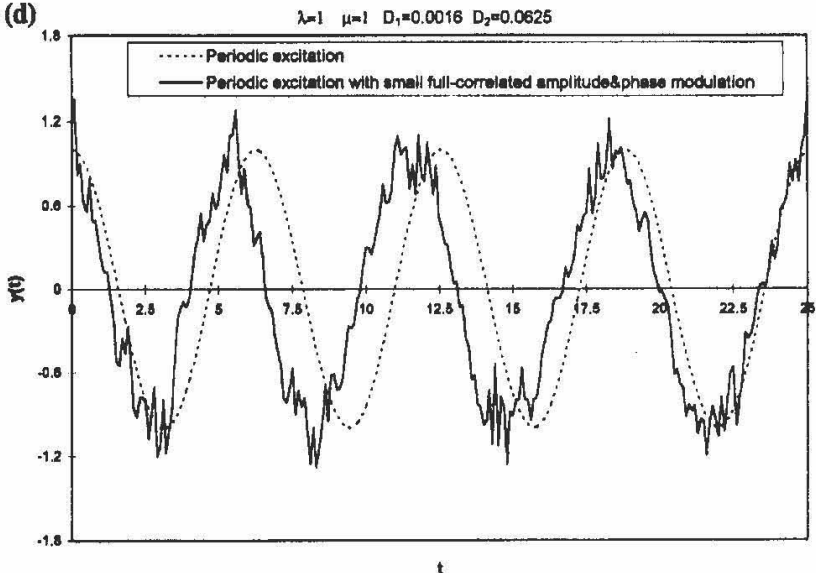

FIG. 1. Periodic Excitation with: (a) Small Phase Modulation; (b) Small Amplitude Modulation; (c) Uncorrelated Small Amplitude and Phase Modulations; (d) Full-Correlated Small Amplitude and Phase Modulations 


$$
z_{3}^{2}+z_{4}^{2}=1
$$

Rewrite (7) in the following format:

$$
d z_{i} / d t=f_{i}+g_{i k} \xi_{k}(t) \quad i=1,2,3,4, \quad k=1,2
$$

where $f_{1}=z_{2} ; f_{2}=-\Omega^{2} z_{1}-2 \alpha z_{2}+\lambda z_{3} ; f_{3}=-\mu z_{4} ; f_{4}=\mu z_{3}$; $g_{21}=z_{3} ; g_{32}=-z_{4} ;$ and $g_{42}=z_{3}$. Because $\xi_{1}(t)$ and $\xi_{2}(t)$ are delta autocorrelated and cross correlated, the correlation times are much smaller than the relaxation time of the system and the drift and diffusion coefficients of a Markov vector approximation can be easily obtained. By appropriately applying the Ito differential rule and the mathematical expectation operator, the deterministic equations for various orders of the response moments can be derived. In this study, after dealing with the extra constraint equations caused by the parent (8), nine independent differential equations for the second-order moments and 25 independent differential equations for the fourth-order moments are finally obtained. These equations may be arranged in matrix forms as

$$
\begin{gathered}
\operatorname{didt}\left(\mathbf{M}_{\mathrm{II}}\right)=\left(\mathbf{A}_{\mathrm{II}}\right)_{9 \times 9} \mathbf{M}_{\mathrm{II}}+\mathbf{C}_{\mathrm{II}} \\
\operatorname{didt}\left(\mathbf{M}_{\mathrm{IV}}\right)=\left(\mathbf{A}_{\mathrm{IV}}\right)_{25 \times 25} \mathbf{M}_{\mathrm{IV}}+\mathbf{C}_{\mathrm{IV}}
\end{gathered}
$$

where $\mathbf{M}_{11}$ and $\mathbf{M}_{\mathrm{IV}} ;\left(\mathbf{A}_{11}\right)_{9 \times 9}$ and $\left(\mathbf{A}_{\mathrm{IV}}\right)_{25 \times 25}$; and $\mathbf{C}_{11}$ and $\mathbf{C}_{\mathrm{IV}}$ are unknown moment vectors, coefficient matrices, and constant column vectors for the second- and fourth-order moments, respectively.

For the second-order moment equation, the moment vector $\mathbf{M}_{\mathrm{II}}$ is defined as

$$
\left(\mathbf{M}_{\mathrm{II}}\right)^{t}=\left\{m_{11}, m_{12}, m_{13}, m_{14}, m_{22}, m_{23}, m_{24}, m_{33}, m_{34}\right\}
$$

where

$$
m_{i j}=E\left[z_{i} z_{j}\right], \quad i=1,2,3 \quad j=1,2,3,4
$$

For the fourth-order moment equation, the moment vector $\mathbf{M}_{\mathrm{IV}}$ is defined as

$$
\begin{gathered}
\left(\mathbf{M}_{\mathrm{IV}}\right)^{t}=\left\{m_{1111}, m_{1112}, m_{1113}, m_{1114}, m_{1122}, m_{1123}, m_{1124}, m_{1133}, m_{1134},\right. \\
m_{1222}, m_{1223}, m_{1224}, m_{1233}, m_{1234}, m_{1333}, m_{1334}, m_{2222}, m_{2223}, m_{2224}, \\
\left.m_{2233}, m_{2234}, m_{2333}, m_{2334}, m_{3333}, m_{3334}\right\}
\end{gathered}
$$

where

$m_{i j k l}=E\left[z_{i} z_{j} z_{k} z_{1}\right], \quad i=1,2,3 \quad j=1,2,3 \quad k=1,2,3$

$$
l=1,2,3,4
$$

The non-zero elements of the coefficient matrices $\mathbf{A}_{\text {II }}$ and $\mathbf{A}_{\text {IV }}$; and constant columns $\mathbf{C}_{\mathrm{II}}$ and $\mathbf{C}_{\mathrm{IV}}$, are listed in Appendix I.

Since only the stationary response is considered in the present study, the time derivatives of the moments in (10) are set to be zero. Thus, a set of linear algebraic equations for the moments are obtained and can be numerically solved.

In the present study, the following nondimensional parameters are used to characterize the system response: $\alpha / \Omega=$ critical damping ratio of the system; $D_{1} \Omega / \lambda^{2}=$ normalized intensity of amplitude fluctuation; $D_{2} / \alpha=$ normalized intensity of phase disturbance; and $\Delta / \alpha=\left(\mu^{2}-\Omega^{2}\right) / 2 \mu \alpha=$ the relative detuning between the natural frequency of the system and the expected frequency of the excitation.

There are two extreme cases. As $D_{1} \Omega / \lambda^{2} \rightarrow 0$ and $D_{2} / \alpha \rightarrow$ 0 , which is the case where random amplitude and phase modulation vanishes, the response of the system reduces to a perfect periodic process because the excitation is a deterministic harmonic function of time. The second extreme case is $D_{1} \Omega /$ $\lambda^{2} \rightarrow 0$ and $D_{2} / \alpha \rightarrow \infty$, implying that the random phase modulation is dominant. An increase of $D_{2}$ generally distributes energy of the excitation more evenly among frequencies and (a)

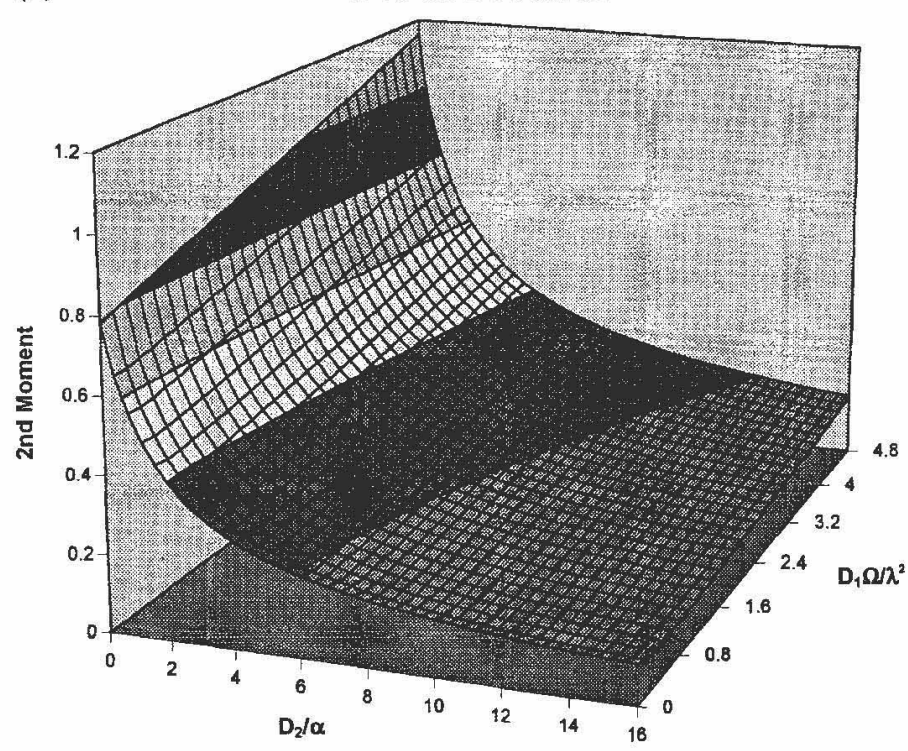

(b)

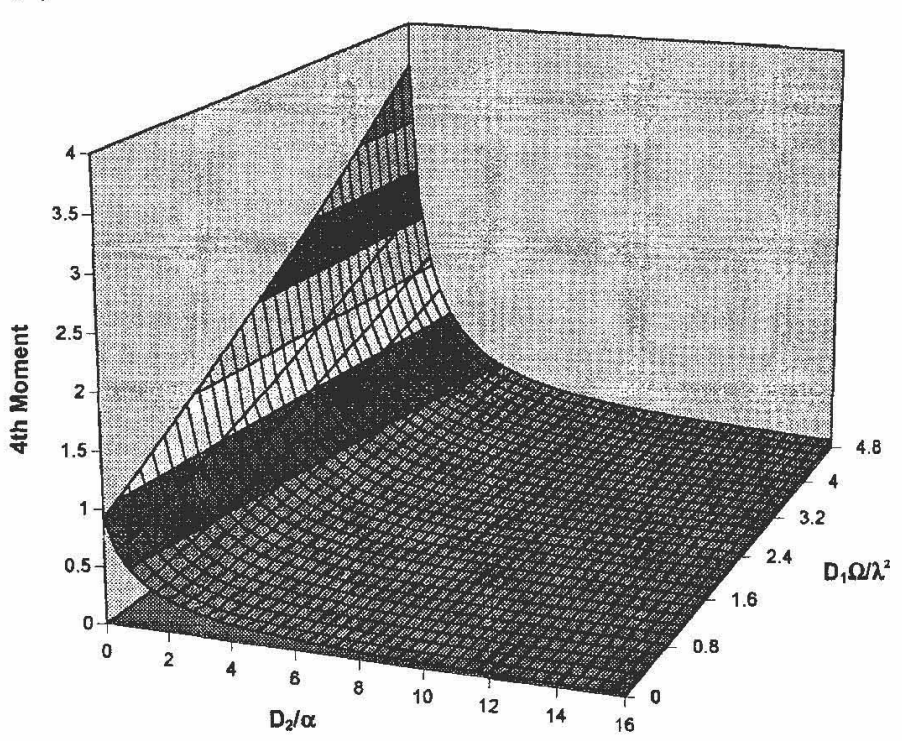

(c) $\quad \Omega=1 \lambda=0.25 \Delta / \alpha=0 \alpha / \Omega=0.1$

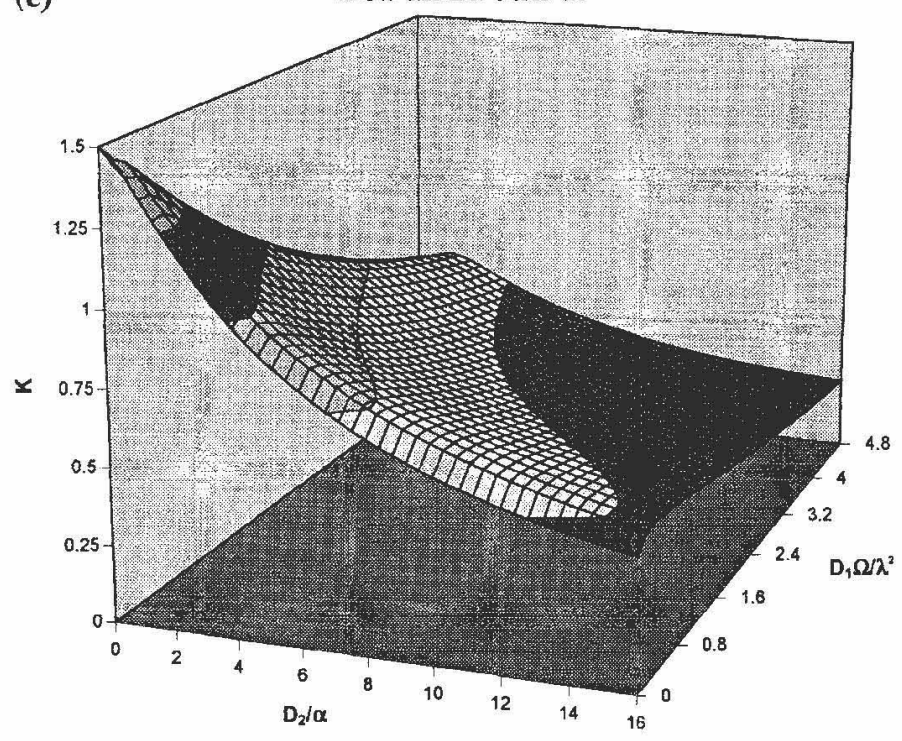

FIG. 2. Second-Order Moment as Function of: (a) $D_{1} \Omega / \lambda^{2}$ and $D_{2} / \alpha(\rho=1)$; (b) $D_{1} \Omega / \lambda^{2}$ and $D_{2} / \alpha(\rho=1)$; (c) $D_{1} \Omega / \lambda^{2}$ and $D_{2} / \alpha(\rho$ $=1$ ) 

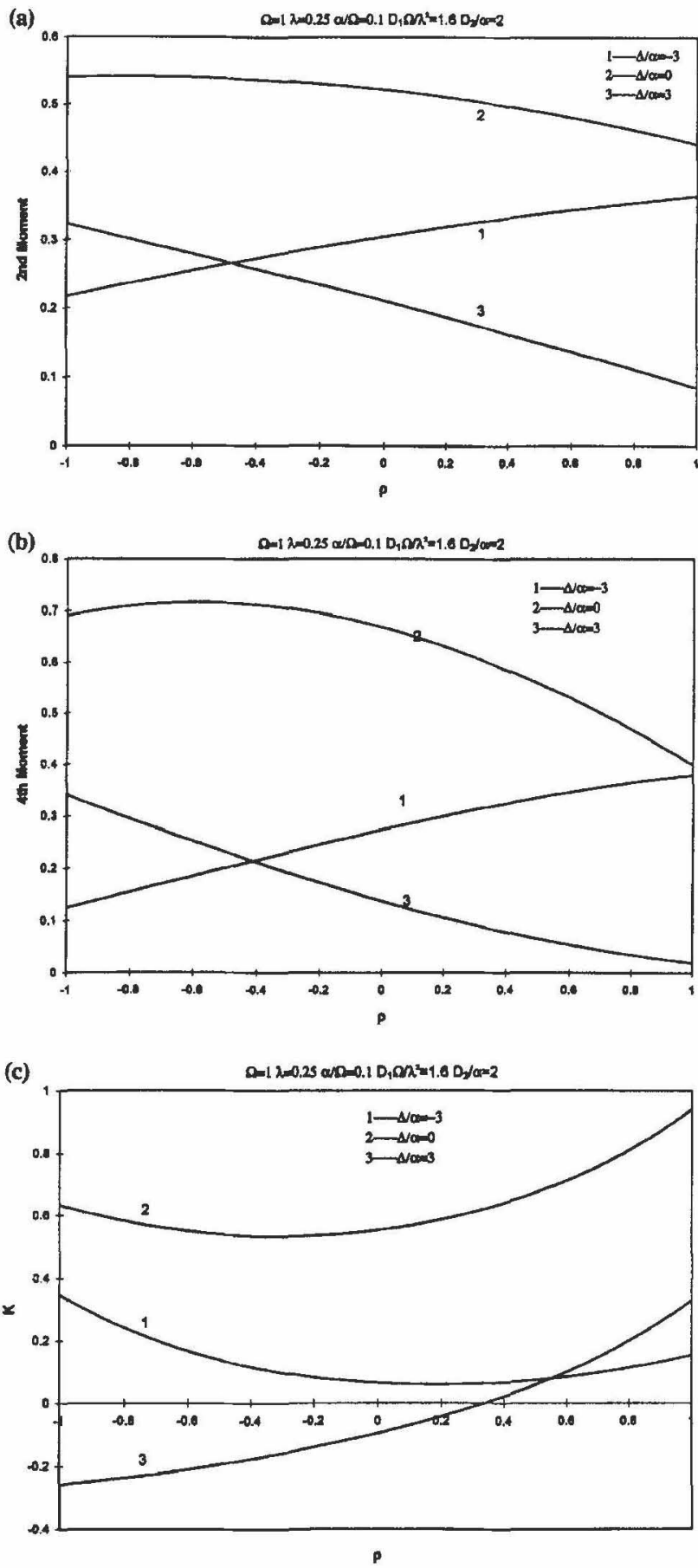

FIG. 3. Function of $\rho$ with Different $\Delta / \alpha$ for: (a) Second-Order Moment; (b) Fourth-Order Moment; (c) Excess Factor

the excitation finally becomes a Delta-correlated white-noisetype process as $D_{2}$ approaches infinity. By the central-limit theorem, the response of a linear SDOF system to such an excitation becomes Gaussian, which is the so-called normalization effect. As results, we have

$$
\operatorname{Lim}_{\substack{D_{1} \Omega / \lambda^{2} \rightarrow 0 \\ D_{2} / \alpha \rightarrow 0}} K=1.5 \operatorname{Lim}_{\substack{D_{1} / \wedge^{2} \rightarrow 0 \\ D_{2} / \alpha \rightarrow \infty}} K=0
$$

The preceding asymptotic properties may be used as a benchmark to verify the numerical results.

Once the moments of the response are obtained, the probability density function (PDF) of the response can be calculated by using the so-called cumulant-neglect closure method (Zeman 1972; Wu and Lin 1984). The characteristic function of the response can be generally expressed as

$$
P_{X}(f)=\operatorname{EXP}\left[\sum_{i=1}^{\infty} \frac{(i 2 \pi f)^{l}}{l !} \kappa_{l}(x)\right]
$$

where $i=$ imaginary unit and $\kappa_{l}(X)$ is the $i$ th order cumulant of a random variable $X . \kappa_{l}(X)$ is defined as

$$
k_{l}(X)=1 /(2 p i)^{l} d^{l} /\left.d f^{l} P_{X}(f)\right|_{f=0}
$$

which may be easily evaluated by moments of the response of up to the $i$ th order. In (17), $d^{l} / d f^{l}$ denotes $l$ th order derivative with respect to the variable $f$. By assuming that cumulants higher than a certain order for non-Gaussian processes vanish, an approximate characteristic function is obtained by using finite terms in (16). Then, the PDF of the response can be calculated by taking the inverse Fourier transform of the characteristic function. In the present study, cumulants higher than fourth order are neglected.
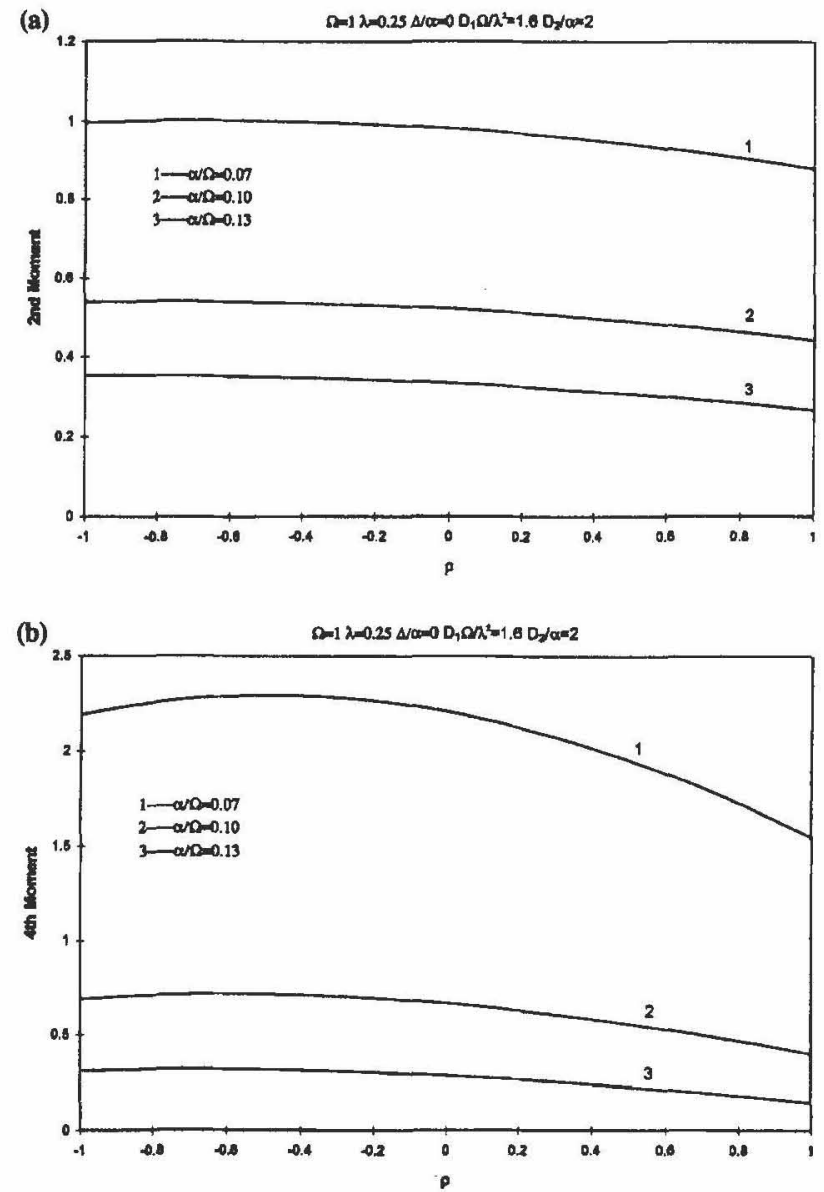

(c)

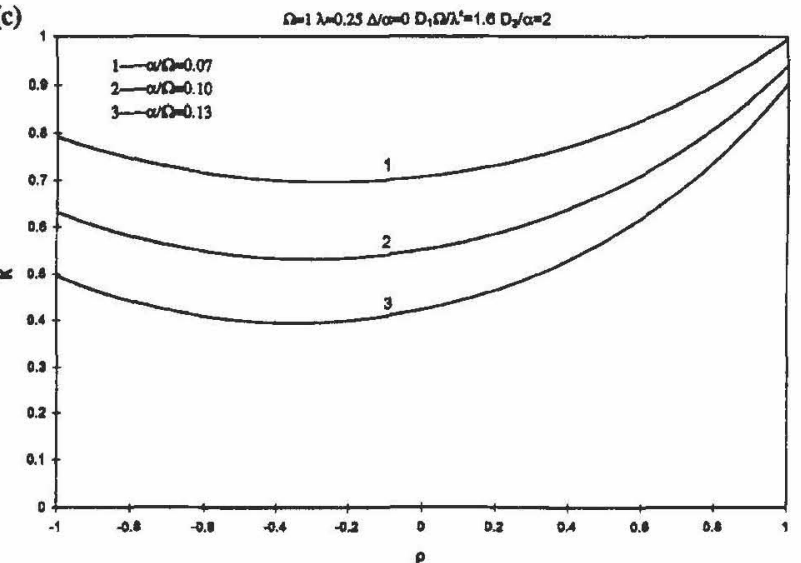

FIG. 4. Function of $\rho$ with Different $\alpha / \Omega$ for: (a) Second-Order Moment; (b) Fourth-Order Moment; (c) Excess Factor 


\section{NUMERICAL RESULTS}

To illustrate applications of the proposed model, numerical results for stationary moment responses of linear SDOF systems subjected to a periodic excitation with correlated random amplitude and phase disturbances are presented. Non-Gaussian nature of the response is investigated in terms of the excess factor as well as the probability density function of the response.

Fig. 2 presents an overall view of the second- and fourthorder moments as well as the excess factor of the stationary response of a lightly damped system at resonance as functions of $D_{1} \Omega / \lambda^{2}$ and $D_{2} / \alpha$ for the case where the random disturbances in amplitude and phase are fully correlated $(\rho=1)$. The second-order moment, fourth-order moment, and excess factor of the response are plotted in Fig. 2(a)-(c), respectively.

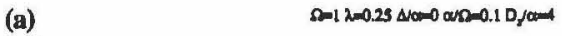

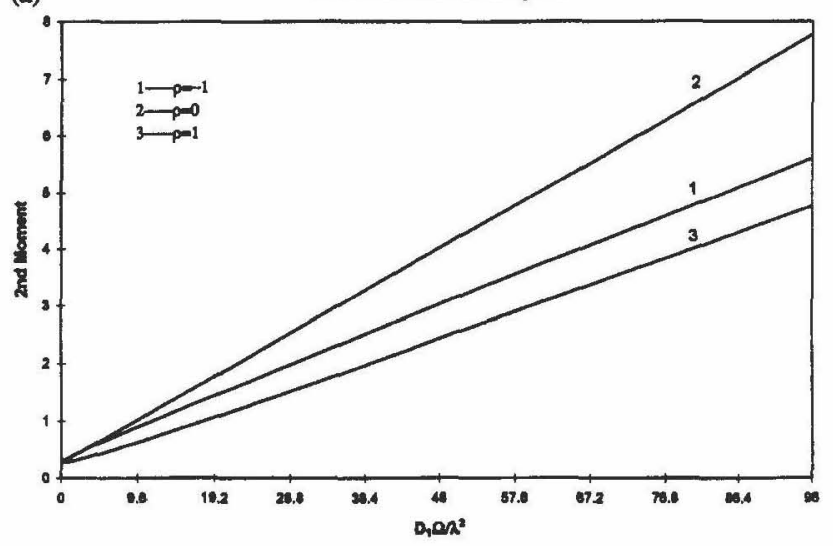

(b) $\quad 0=1 \lambda=0.23 \Delta / \alpha=0,0 / 0-0.10,100=1$

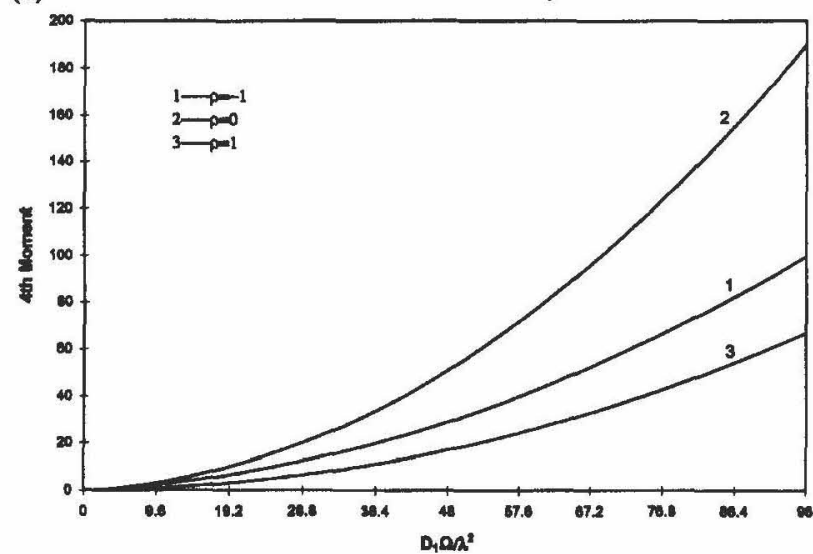

(c)

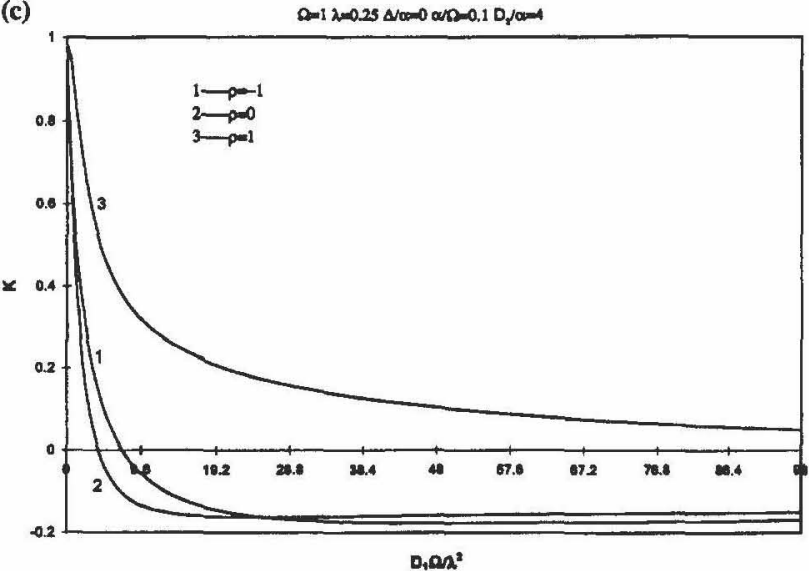

FIG. 5. Function of $D_{1} \Omega / \lambda^{2}$ with Different $\rho$ for: (a) Second-Order Moment; (b) Fourth-Order Moment; (c) Excess Factor
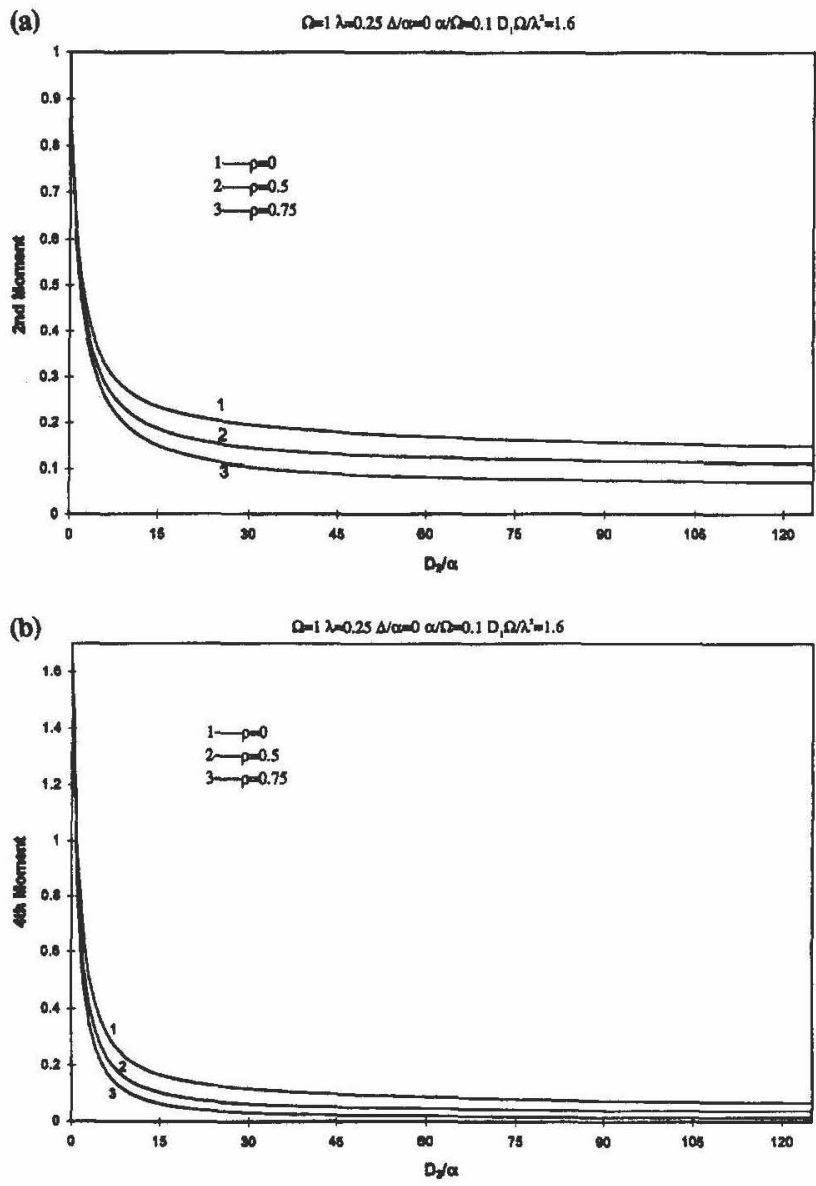

(c)

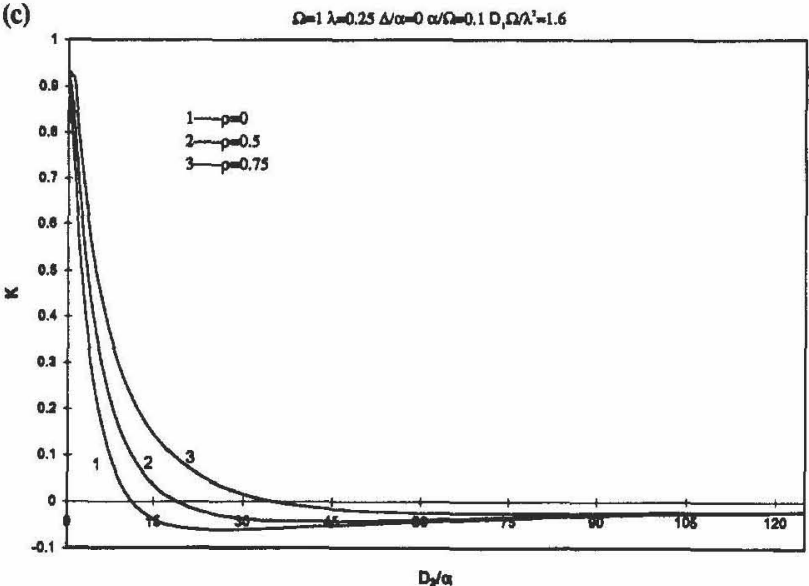

FIG. 6. Function of $D_{2} / \alpha$ with Different $p$ for: (a) Second-Order Moment; (b) Fourth-Order Moment; (c) Excess Factor

It is observed that increasing amplitude fluctuation level, $D_{1}$, results in larger second- and fourth-order moment responses, while increasing phase modulation level, i.e., $D_{2}$, generally causes smaller moment responses. The latter may be attributed to the normalization effect. The excess factor, $K$, achieves the value of 1.5 , as expected, when $D_{1} \Omega / \lambda^{2}$ and $D_{2} / \alpha$ are both equal to zero. As compared with the case of a perfect periodic excitation, i.e., $D_{1}=D_{2}=0$, the numerical results show that existence of the random disturbance in either amplitude or phase significantly changes the moment responses. Trends and variations of the response may be more clearly seen by twodimensional curves such as those presented in later in this paper.

The influence of $\rho$ can be observed from Figs. 3 and 4 , where the moment responses and the excess factor are plotted 

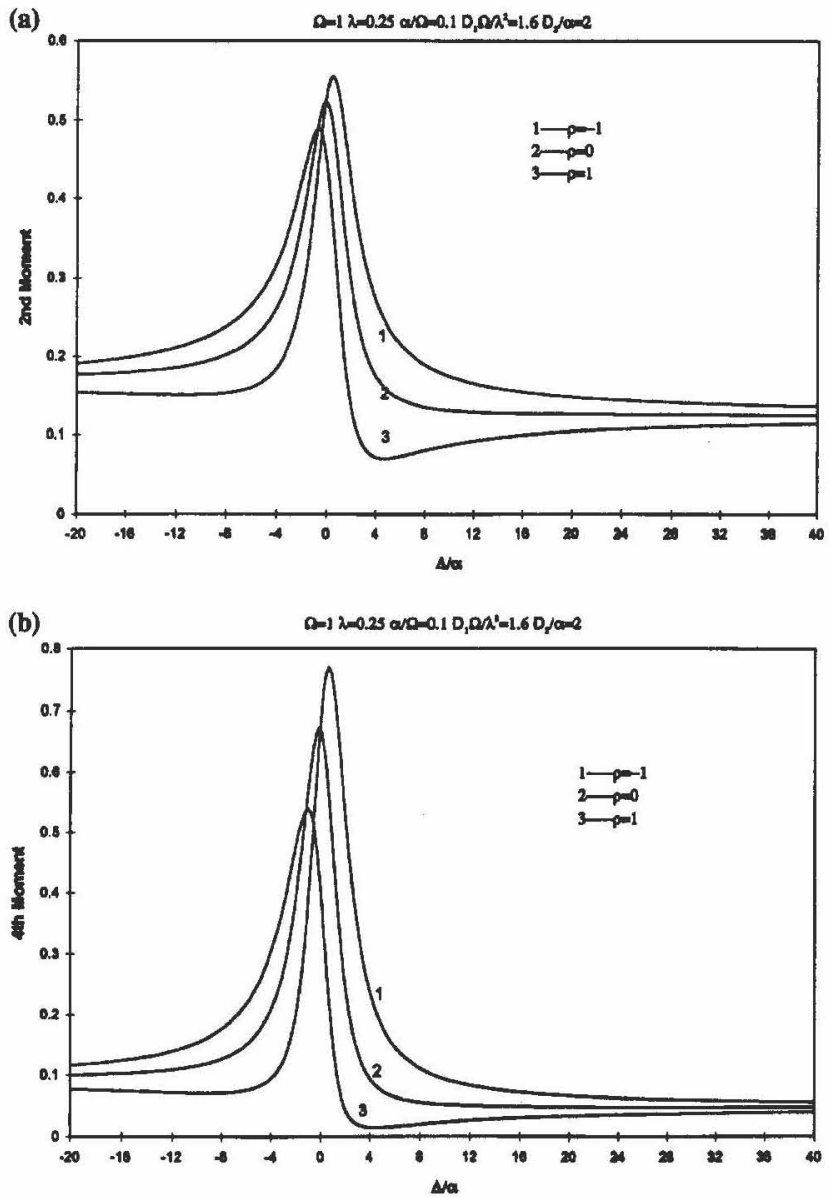

(c)

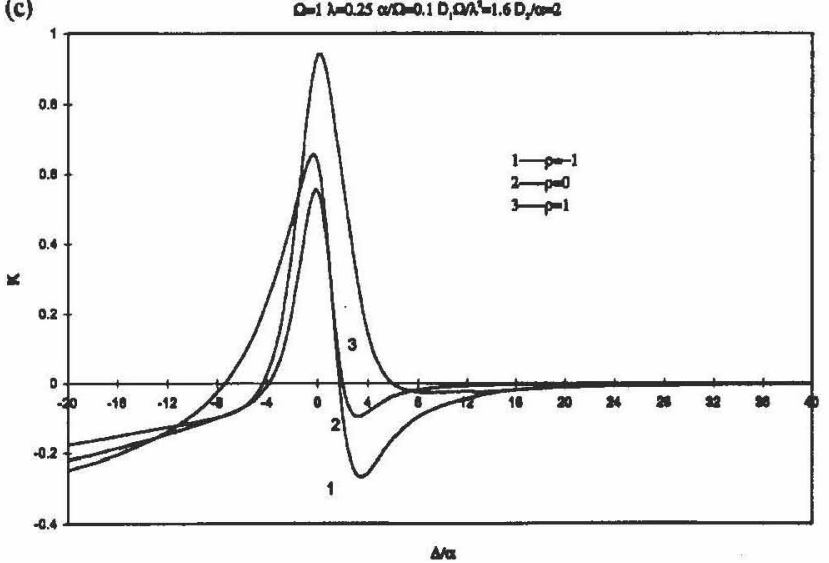

FIG. 7. Function of $\Delta / \alpha$ with Different $p$ for: (a) Second-Order Moment; (b) Fourth-Order Moment; (c) Excess Factor

versus $\rho$ directly, using the detuning and the critical damping ratio as parameters, respectively. The results in Fig. 3 illustrate that positive and negative detuning may have different effects on the system response. As observed in Fig. 3, the moment responses grow with $p$ for the case of negative detuning $(\Delta /$ $\Omega=-3$, i.e., $\mu<\Omega$ ), but they monotonically decrease with $\rho$ in the range of $-1 \leq \rho \leq 1$ for positive detuning $(\Delta / \Omega=$ 3 , i.e., $\mu>\Omega)$. In the case of resonance $(\Delta / \Omega=0$, i.e., $\mu=$ $\Omega$ ), the moment responses are higher than those for the case of nonresonance. As seen in Fig. 4, increasing damping coefficient $\alpha$ reduces system moment responses, which is expected. In both figures, all curves are not symmetric about $\rho$ $=0$, indicating that positive and negative correlation may have different effects on the system response.

The moment responses and the excess factor are plotted as a function of $D_{1} \Omega / \lambda^{2}$ for different values of $\rho$ and a fixed value of $D_{2} / \alpha$ in Fig. 5. The moments increase with $D_{1} \Omega / \lambda^{2}$, while the excess factor decreases rapidly with $D_{1} \Omega / \lambda^{2}$ in the beginning and changes slowly afterwards. For very large values of $D_{1} \Omega / \lambda^{2}$, further calculations show that the excess factor converges to a limiting value. It is also observed that there exists a linear relationship between the second-order moment response and $D_{1}$ [Fig. 5(a)].

Fig. 6 is similar to Fig. 5 except the results are presented as a function of $D_{2} / \alpha$ for a fixed value of $D_{1} \Omega / \lambda^{2}$. Large-phase modulation reduces the system moment response. For extremely large $D_{2} / \alpha$, the system response including the moments and the excess factor converges, due to an averaging effect, to a limiting value depending on $D_{1} \Omega / \lambda^{2}$. In fact, for the case of $D_{1} \Omega / \lambda^{2}=0$, the excess factor converges to
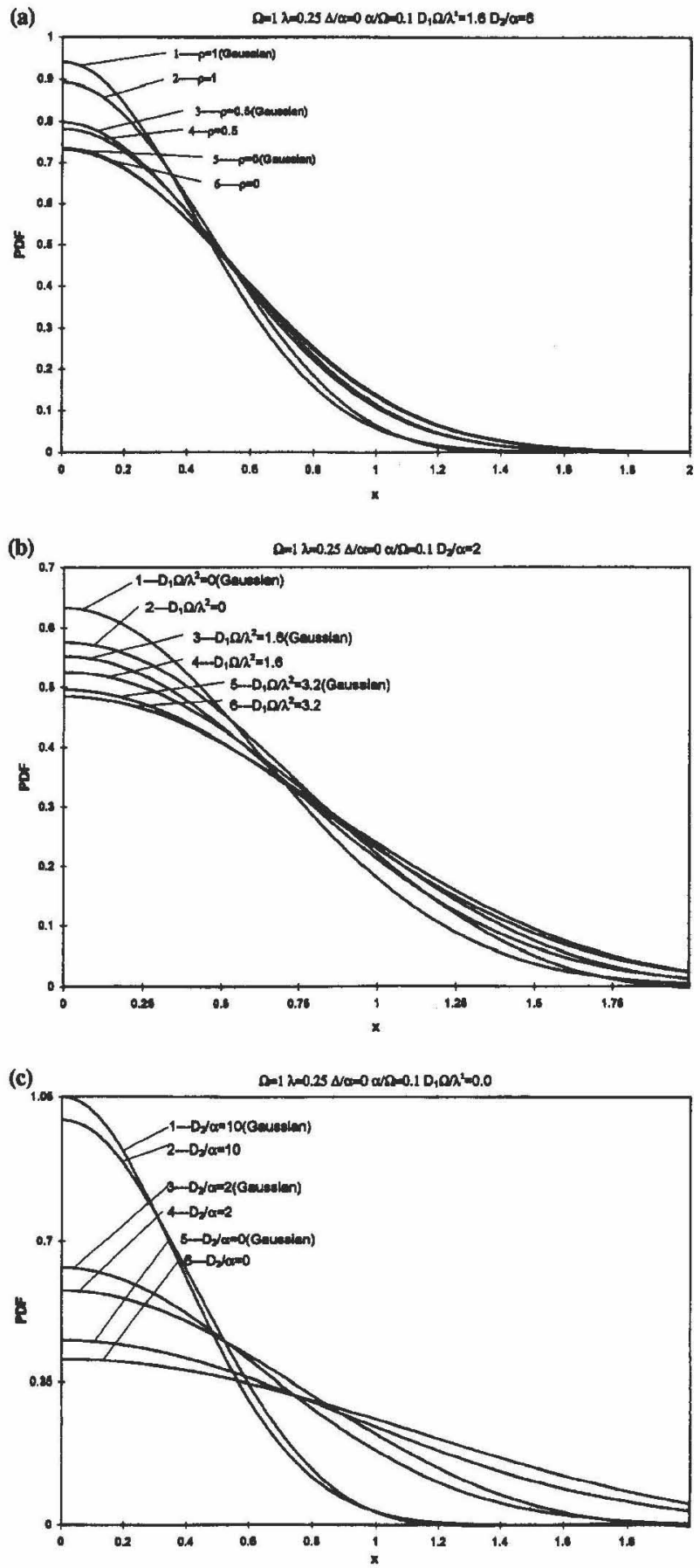

FIG. 8. Probability Density Functlon with Different: (a) $\rho$; (b) $D_{1} \Omega / \lambda^{2}(\rho=0) ;(c) D_{2} / \alpha(\rho=0)$ 
zero - the value of Gaussian process - which suggests that the response may become Gaussian for sufficiently large random phase disturbances at least in the sense of up to the fourthorder moment (Dimentberg et al. 1993).

The role of relative detuning between the system natural frequency $\Omega$ and the mean excitation frequency $\mu$ can be seen in Fig. 7. Curves of the second- and the fourth-order moments and the excess factor versus the detuning factor, $\Delta / \alpha$, for fixed $D_{1} \Omega / \lambda^{2}, D_{2} / \alpha$ and three different levels of $\rho$ are illustrated. There is a peak observed near the resonance for each individual curve and the position of the peak slightly changes depending on the $\rho$-value. For a small difference of relative detuning away from the peak position, the moment responses decrease rapidly, as expected. For large detuning, however, the moment responses changes slowly with $\Delta / \alpha$. In the case of very large positive detuning $(\mu \gg \Omega$ ), the excess factors with different $\rho$ all converge to zero, which is the value for Gaussian process. Larger $\rho$ will in general reduce resonance moment responses and peak frequency. It is also noticed that the excess factor, $K$, may become zero for some specific detuning values. In other words, for some particular combinations of parameters, the response of the system under non-Gaussian excitation may demonstrate some Gaussian nature, as far as only the moments not higher than the fourth order are concerned.

The probability density functions (PDF) of system response with different levels of $\rho, D_{1} \Omega / \lambda^{2}$ and $D_{2} / \alpha$ are plotted in Fig. 8. For comparison, the corresponding Gaussian distribution curves are also obtained by assuming that moments higher than second order are zero. For larger positive cross-correlation coefficient between $\xi_{1}(t)$ and $\xi_{2}(t)$, the distribution is further away from that of Gaussian distribution and its peak value at $x=0$ is higher, as shown in Fig. 8(a). As $D_{1} \Omega / \lambda^{2}$ increases [Fig $8(\mathrm{~b})$ ], the curve becomes flatter and so that the moment response gets larger. For large $D_{2} / \alpha$ [Fig. 8(c)], the differences between the PDF of the response and the corresponding normal distribution clearly indicate the non-Gaussian nature of the response. The PDF curve becomes steeper with larger $D_{2} /$ $\alpha$, which leads to smaller moment responses. It should be mentioned that the cumulant-neglect closure method yields some negative PDF values (in the order of $10^{-3}$ or less in this study) for relatively large $x$, and, therefore, should be cautious when the results are applied for estimating exceedance probabilities of high threshold values, as indicated in the literature (Schueller and Bucher 1988).

\section{SUMMARY AND CONCLUSIONS}

A stochastic model for periodic excitations with correlated random amplitude and phase disturbances is presented. Application of this model is demonstrated by stationary moment responses of a linear SDOF system subject to such an excitation. Numerical results for the second- and fourth-order moments are illustrated to show the effects of the intensities and correlation of the random disturbances, relative detuning, and viscous damping on the system response. The non-Gaussian nature of the response is investigated in terms of the excess factor as well as the probability density function. The conclusions drawn from this study are summarized as follows:

1. The correlation between the random disturbances in amplitude and phase of periodic excitations significantly affects the system moment response and, therefore, should be taken into account in the analysis.

2. For a lightly damped system at resonance, large amplitude disturbance results in large system moment response and larger phase modulation may reduce moment response. For very large random phase modulation, the response converges to limiting value depending on the intensities and correlation between the random disturbances, and, in the case of phase modulation alone, the response becomes Gaussian due to the normalization effect.

3. The system moment response depends on detuning parameter $\Delta / \alpha$. Correlation between random disturbances affects the peak response and the peak frequency of curves of the moments versus the relative detuning. For a sufficiently large positive relative detuning $(\mu>\Omega)$, the response process becomes close to a Gaussian process in the sense of up to the fourth-order moment, and, therefore, information on higher-order moments may be needed for investigation of the non-Gaussian nature of the response.

4. Larger damping coefficient leads to reduction of the moment responses in both cases of uncorrelated and correlated random disturbances.

\section{ACKNOWLEDGMENT}

This research is partly supported by ONR-URI under Grant No. N00014-93-1-0917. This support is gratefully acknowledged by the writers.

\section{APPENDIX I. MATRICES ELEMENTS}

The non-zero elements of the coefficient matrix $\mathbf{A}_{\text {II }}$ are

$$
\begin{aligned}
& a_{12}=2, \quad a_{21}=-\Omega^{2}, \quad a_{22}=-2 \alpha, \quad a_{23}=\lambda, \quad a_{24}=-(1 / 2) D_{12}, \\
& a_{25}=1, \quad a_{33}=-(1 / 2) D_{2}, \quad a_{34}=-\mu, \quad a_{36}=1, \quad a_{43}=\mu, \\
& a_{44}=-(1 / 2) D_{2}, \quad a_{47}=1, \quad a_{52}=-2 \Omega^{2}, \quad a_{55}=-4 \alpha, \\
& a_{56}=2 \lambda, \quad a_{57}=-D_{12}, \quad a_{58}=D_{1}, \quad a_{63}=-\Omega^{2}, \\
& a_{66}=-\left[2 \alpha+(1 / 2) D_{2}\right], \quad a_{67}=-\mu, \quad a_{68}=\lambda, \quad a_{69}=(3 / 2) D_{12}, \\
& a_{74}=-\Omega^{2}, \quad a_{76}=\mu, \quad a_{77}=-\left[2 \alpha+(1 / 2) D_{2}\right], \\
& a_{78}=(3 / 2) D_{12}, \quad a_{79}=\lambda, \quad a_{88}=-2 D_{2}, \quad a_{89}=-2 \mu, \\
& a_{98}=2 \mu, \quad a_{99}=-2 D_{2}
\end{aligned}
$$

The non-zero elements of constant column $\mathbf{C}_{11}$ are

$$
c_{7}=-(1 / 2) D_{12}, \quad c_{8}=D_{2}, \quad c_{9}=-\mu
$$

The non-zero elements of coefficient matrix $\mathbf{A}_{\mathrm{IV}}$ are

$$
\begin{aligned}
& \hat{a}_{1,2}=4, \quad \hat{a}_{2,1}=-\Omega^{2}, \quad \hat{a}_{2,2}=-2 \alpha, \quad \hat{a}_{2,3}=\lambda, \quad \hat{a}_{2,4}=-(1 / 2) D_{12}, \\
& \hat{a}_{2,5}=3, \quad \hat{a}_{3,3}=-(1 / 2) D_{2}, \quad \hat{a}_{3,4}=-\mu, \quad \hat{a}_{3,6}=3, \quad \hat{a}_{4,3}=\mu, \\
& \hat{a}_{4,4}=-(1 / 2) D_{2}, \quad \hat{a}_{4,7}=3, \quad \hat{a}_{5,2}=-2 \Omega^{2}, \quad \hat{a}_{5,5}=-4 \alpha, \\
& \hat{a}_{5,6}=2 \lambda, \quad \hat{a}_{5,7}=-D_{12}, \quad \hat{a}_{5,8}=D_{1}, \quad \hat{a}_{5,10}=2, \quad \hat{a}_{6,3}=-\Omega^{2}, \\
& \hat{a}_{6,6}=-\left[\begin{array}{ll}
2 \alpha+(1 / 2) D_{2} &
\end{array}, \quad \hat{a}_{6,7}=-\mu, \quad \hat{a}_{6,8}=\lambda,\right. \\
& \hat{a}_{6,9}=-(3 / 2) D_{12}, \quad \hat{a}_{6,11}=2, \quad \hat{a}_{7,4}=-\Omega^{2}, \quad \hat{a}_{7,6}=\mu \\
& \hat{a}_{7,7}=-\left[2 \alpha+(1 / 2) D_{2}\right], \quad \hat{a}_{7,8}=(3 / 2) D_{12}, \quad \hat{a}_{7,9}=\lambda, \\
& \hat{a}_{7,12}=2, \quad \hat{a}_{8,8}=-2 D_{2}, \quad \hat{a}_{8,9}=-2 \mu_{2}, \quad \hat{a}_{8,13}=2, \quad \hat{a}_{9,8}=2 \mu_{2}, \\
& \hat{a}_{9,9}=-2 D_{2}, \quad \hat{a}_{9,14}=2, \quad \hat{a}_{10,5}=-3 \Omega^{2}, \quad \hat{a}_{10,10}=-6 \alpha, \\
& \hat{a}_{10,11}=3 \lambda, \quad \hat{a}_{10,12}=-(3 / 2) D_{12}, \quad \hat{a}_{10,13}=3 D_{1}, \quad \hat{a}_{10,17}=1, \\
& \hat{a}_{11,6}=-2 \Omega^{2}, \quad \hat{a}_{11,11}=-\left[4 \alpha+(1 / 2) D_{2}\right], \quad \hat{a}_{11,12}=-\mu, \\
& \hat{a}_{11,13}=2 \lambda, \quad \hat{a}_{11,14}=-3 D_{12}, \quad \hat{a}_{11,15}=D_{1}, \quad \hat{a}_{11,18}=1, \\
& \hat{a}_{12,7}=-2 \Omega^{2}, \quad \hat{a}_{12,11}=\mu, \quad \hat{a}_{12,12}=-\left[4 \alpha+(1 / 2) D_{2}\right], \\
& \hat{a}_{12,13}=3 D_{12}, \quad \hat{a}_{12,14}=2 \lambda, \quad \hat{a}_{12,16}=D_{1}, \quad \hat{a}_{12,19}=1, \\
& \hat{a}_{13,8}=-\Omega^{2}, \quad \hat{a}_{13,13}=-2\left(\alpha+D_{2}\right), \quad \hat{a}_{13,14}=-2 \mu, \quad \hat{a}_{13,15}=\lambda,
\end{aligned}
$$




$$
\begin{aligned}
& \hat{a}_{13,16}=-(5 / 2) D_{12}, \quad \hat{a}_{13,20}=1, \quad \hat{a}_{14,9}=-\Omega^{2}, \quad \hat{a}_{14,13}=2 \mu, \\
& \hat{a}_{14,14}=-2\left(\alpha+D_{2}\right), \quad \hat{a}_{14,15}=(5 / 2) D_{12}, \quad \hat{a}_{14,16}=\lambda, \quad \hat{a}_{14,21}=1, \\
& \hat{a}_{15,15}=-(9 / 2) D_{2}, \quad \hat{a}_{15,16}=-3 \mu, \quad \hat{a}_{15,22}=1, \quad \hat{a}_{16,15}=3 \mu, \\
& \hat{a}_{16,16}=-(9 / 2) D_{2}, \quad \hat{a}_{16,23}=1, \quad \hat{a}_{17,10}=-3 \Omega^{2}, \quad \hat{a}_{17,17}=-8 \alpha, \\
& \hat{a}_{17,18}=4 \lambda, \quad \hat{a}_{17,19}=-2 D_{12}, \quad \hat{a}_{17,19}=6 D_{1}, \quad \hat{a}_{18,11}=-3 \Omega^{2}, \\
& \hat{a}_{18,18}=-\left[6 \alpha+(1 / 2) D_{2}\right], \quad \hat{a}_{18,19}=-\mu, \quad \hat{a}_{18,20}=3 \lambda, \\
& \hat{a}_{18,21}=-(9 / 2) D_{12}, \quad \hat{a}_{18,22}=3 D_{1}, \quad \hat{a}_{19,12}=-3 \Omega^{2}, \quad \hat{a}_{19,18}=\mu, \\
& \hat{a}_{19,19}=-\left[6 \alpha+(1 / 2) D_{2}\right], \quad \hat{a}_{19,20}=(9 / 2) D_{12}, \quad \hat{a}_{19,21}=3 \lambda, \\
& \hat{a}_{19,23}=3 D_{1}, \quad \hat{a}_{20,13}=-2 \Omega^{2}, \quad \hat{a}_{20,20}=-\left(4 \alpha+2 D_{2}\right), \\
& \hat{a}_{20,21}=-2 \mu, \quad \hat{a}_{20,22}=2 \lambda, \quad \hat{a}_{20,23}=-5 D_{12}, \quad \hat{a}_{20,24}=D_{1}, \\
& \hat{a}_{21,14}=-2 \Omega^{2}, \quad \hat{a}_{21,20}=2 \mu, \quad \hat{a}_{21,21}=-\left(4 \alpha+2 D_{2}\right), \\
& \hat{a}_{21,22}=5 D_{12}, \quad \hat{a}_{21,23}=2 \lambda, \quad \hat{a}_{21,25}=D_{1}, \quad \hat{a}_{22,15}=-\Omega^{2}, \\
& \hat{a}_{22,22}=-\left[2 \alpha+(9 / 2) D_{2}\right], \quad \hat{a}_{22,23}=-3 \mu, \quad \hat{a}_{22,24}=\lambda, \\
& \hat{a}_{22,25}=-(7 / 2) D_{12}, \quad \hat{a}_{23,16}=-\Omega^{2}, \quad \hat{a}_{23,22}=3 \mu, \\
& \hat{a}_{23,23}=-\left[2 \alpha+(9 / 2) D_{2}\right], \quad \hat{a}_{23,23}=(7 / 2) D_{12}, \quad \hat{a}_{23,25}=\lambda, \\
& \hat{a}_{24,24}=-8 D_{2}, \quad \hat{a}_{24,25}=-4 \mu, \quad \hat{a}_{25,24}=4 \mu, \quad \hat{a}_{25,25}=-8 D_{2}
\end{aligned}
$$

The non-zero elements of constant column $\mathbf{C}_{\mathrm{IV}}$ are

$$
\begin{aligned}
& \hat{c}_{7}=-(1 / 2) D_{12} m_{11}, \quad \hat{c}_{8}=D_{2} m_{11}, \quad \hat{c}_{9}=-\mu m_{11}, \quad \hat{c}_{12}=-D_{12} m_{12}, \\
& \hat{c}_{13}=D_{2} m_{12}, \quad \hat{c}_{14}=-\mu m_{12}, \quad-(3 / 2) D_{12} m_{13}, \quad \hat{c}_{15}=3 D_{2} m_{13}, \\
& \hat{c}_{16}=D_{2} m_{14}-2 \mu m_{13}, \quad \hat{c}_{19}=-(3 / 2) D_{12} m_{22}, \quad \hat{c}_{20}=D_{22} m_{22}, \\
& \hat{c}_{21}=-\mu m_{22}-2 D_{12} m_{23}, \quad \hat{c}_{22}=2 D_{22} m_{23}, \\
& \hat{c}_{23}=D_{2} m_{24}-2 \mu m_{23}-(5 / 2) D_{12} m_{33}, \quad \hat{c}_{24}=6 D_{2} m_{33}, \\
& \hat{c}_{25}=3 D_{2} m_{34}-3 \mu m_{33}
\end{aligned}
$$

\section{APPENDIX II. REFERENCES}

Dimentberg, M. G. (1988). Statistical dynamics of nonlinear and timevarying system. Research Studies Press Ltd., Letchworth, England.

Dimentberg, M. F. (1991). "A stochastic model of parametric excitation of a straight pipe due to slug flow of a two-phase fluid." Proc., 5 th Int. Conf. on Flow-Induced Vibrations, Brighton, England, 207-209.

Dimentberg, M. F. (1992). "Stability and subcritical dynamics of structures with spatially disordered traveling parametric excitation." Probabilistic Engrg. Mech., 7, 131-134.

Dimentberg, M. F., Hou, Z. K., Noori, M. N., and Zhang, W. (1993). "Non-Gaussian response of a single-degree-of-freedom system to a periodic excitation with random phase modulation." Recent development in the mechanics of continua (special volume). ASME, New York, N.Y.

Grigoriu, M., Ruiz, S. E., and Rosenblueth, E. (1988), "Nonstationary models of seismic ground acceleration." Earthquake Spectra, 4(3), 551-568.

Hou, Z. K., Zhou, Y. S., Dimentberg, M. F., and Noori, M. (1994), "Non- stationary non-Gaussian response of linear SDOF system under randomly disordered periodic excitation." Proc., Int. Conf. on Vibration Engrg., Beijing, China, 415-420.

Hou, Z. K., Zhou, Y. S., Dimentberg, M. F., and Noori, M. (1995). "A non-stationary stochastic model for periodic excitation with random phase modulation." Prababilistic Engrg. Mech., 10(1995), 73-81.

Hou, Z. K., Zhou, Y. S., Dimentberg, M. F., and Noori, M. (1995). "A stationary model for periodic excitations with uncorrelated random disturbances." Proc., 3rd Stochastic Struct. Dynamics Conf., San Juan, Puerto Rico, Jan. 15-18, 1995.

Lin, Y. K., and Li, Q. C. (1993). "New stochastic theory for bridge stability in turbulent flow." J. Engrg. Mech., ASCE, 119, 113-127.

Longuet-Higgins, M. S. (1957). "The statistical analysis of a random, moving surface." Philosophical Trans. Royal Soc., London, England, Ser. A(966), 321-387.

Schueller, G. I., and Bucher, C. G. (1988). "Non-Gaussian response of systems under dynamic excitation." Stochastic structural dynamics (progress in theory and applications), S. T. Ariaratnam, G. I. Schueller, and I. Elishakoff, eds., 219-239.

Shinozuka, M., and Deodatis, G. (1988). "Stochastic process models for earthquake ground motion." Probabilistic Engrg. Mech., 3(3), 114123.

Wedig, W. (1989), "Analysis and simulation of nonlinear stochastic systems." Nonlinear Dynamics in Engineering Systems, W. Schiehlen, ed., Springer-Verlag KG, Berlin, Germany, 337-344.

Wu, W. F., and Lin, Y. K. (1984). "Cumulant neglect closure for nonlinear oscillators under random parametric and external excitation." Int. J. Non-linear Mech., 19, 349-362.

Zeman, J. L. (1972). "'Zur losung nichtlinearer stochastischer probleme der mechanik." Acta Mechanica, 14, 157-169.

\section{APPENDIX III. NOTATION}

The following symbols are used in this paper:

$\mathbf{A}_{\mathrm{II}}=$ coefficient matrix for second-order moments;

$\mathbf{A}_{1 v}=$ coefficient matrix for fourth-order moments;

$\mathrm{C}_{\mathrm{II}}=$ constant column for second-order moment;

$\mathrm{C}_{\mathrm{IV}}=$ constant column for fourth-order moment;

$D_{1}=$ intensity of $\xi_{1}(t)$;

$D_{2}=$ intensity of $\xi_{2}(t)$;

$D_{12}=$ cross intensity of $\xi_{1}(t)$ and $\xi_{2}(t)$;

$f_{1}, f_{2}, f_{3}, f_{4}=$ functions of state variables;

$g_{21}, g_{32}, g_{42}=$ parametric coefficients;

$K=$ excess factor;

$\mathbf{M}_{\mathrm{II}}=$ moment vector for second-order moment;

$\mathbf{M}_{\mathrm{IV}}=$ moment vector for fourth-order moment;

$P_{X}(f)=$ characteristic function;

$x(t)=$ displacement response;

$y(t)=$ excitation;

$z_{1}, z_{2}, z_{3}, z_{4}=$ state variables;

$\alpha=$ damping constant of the system;

$\Delta=$ frequency detuning between system and excitation;

$\kappa(x)=$ cumulant of $x$;

$\lambda=$ mean amplitude of excitation;

$\mu=$ mean frequency of excitation;

$\xi_{1}(t)=$ amplitude disturbance (Gaussian white noise);

$\xi_{2}(t)=$ phase disturbance (Gaussian white noise);

$\rho=$ cross correlation coefficient; and

$\Omega=$ natural frequency of the system. 